D) Check for updates

Cite this: Mater. Adv., 2021, 2, 2188

Received 24th January 2021, Accepted 16th February 2021 DOI: 10.1039/d1ma00060h

rsc.li/materials-advances

\title{
Essential role of quantum science and nanoscience in antiviral strategies for COVID-19
}

\author{
Mina Zare, (D) *a Mika Sillanpääb and Seeram Ramakrishna (D) *a
}

\begin{abstract}
The severe acute respiratory syndrome coronavirus (SARS-CoV-2) that caused the COVID-19 disease pandemic since December 2019 highlights the importance of developing efficient antiviral strategies to prevent and treat viral infection. Virus particles are nanosized, therefore design strategies are suitable to develop advanced nano and quantum materials that can interact with and mimic virus particles. Quantum science and nanoscience can help in the synthesis and functionalization of nanomaterials for virus prevention, treatment, and detection. The first step is identifying the genome sequencing of the virus, then the fabrication of antiviral (virustatic or virucidal) materials. Quantum and nanoscienceenabled nanomaterials yield better performance and flexibility for virus detection, vaccine design and delivery, facemasks, respirators, and drugs. In this review, we explore the applications of quantum and nanoscience in diagnosis, inhibition, and antiviral therapy. Further, we explain how the van der Waals forces, electrostatic interactions, fluctuation of the medium, electrodynamic and thermal fluctuations, local field enhancement effect, and reactive oxygen species (ROS) generation can prevent the viral life cycle through the virustatic and virucidal effects of quantum and nanoparticles.
\end{abstract}

\section{Introduction}

In the past 20 years, viral infections such as Ebola, Zika, Middle East respiratory syndrome coronavirus (MERS-CoV), and severe acute respiratory syndrome coronavirus-1 (SARS-CoV1) have caused pandemics and deaths. Infectious diseases are a major threat to global health that we must be aware of, and we must be well prepared to combat future viral pandemics of understudied or unknown viruses. ${ }^{1,2}$ The World Health Organization (WHO) in 2018 prepared a list of non-viral and viral pathogens that could cause Disease $\mathrm{X}$ with epidemic potential for which there is no or insufficient treatment. Severe acute respiratory syndrome coronavirus 2 (SARS-CoV-2) is the most recent pandemic virus and the first Disease X. It causes COVID-19 and was recognized in December 2019 and started in Wuhan, China. To date (23 January 2021), about 2.06 million deaths and 96.2 million cases have been reported. ${ }^{3,4}$ WHO declared a Public Health Emergency of International Concern (PHEIC) on 30 January 2020 and declared the outbreak a pandemic on 11 March 2020. ${ }^{5-7}$

The responses to a pandemic will be different from one context to another, from one society to another, but they must be based on a human rights framework and humanistic vision

\footnotetext{
${ }^{a}$ Center for Nanotechnology and Sustainability, National University of Singapore, Singapore 117576, Singapore.E-mail: seeram@nus.edu.sg, zare.mina@yahoo.com ${ }^{b}$ Chemistry Department, College of Science, King Saud University, Riyadh 11451, Saudi Arabia
}

of education and development. Actions must strengthen public education, fortify common goods, expand global solidarity and emphasize the collective responsibility for the education of everyone everywhere. For that future, we need boldness of thought and courageous action now. ${ }^{8}$ To reduce the potentially devasting effects of COVID-19, immediate support is required from social and behavioral science. Plenty of activities may be relevant to future public health crises and pandemics. The WHO report disclosed that "health communication is seen to have relevance for virtually every aspect of health and well-being, including disease prevention, health promotion and quality of life". 9,10 The SARS-CoV-2 pandemic has ignited scientific efforts to develop quantum science and nanoscience-enabled solutions intended to stop viral infections. The preventive, diagnostic, and therapeutic applications of quantum and nanomaterials have the potential to bring about revolutionary developments in the biomedical field. ${ }^{11}$

SARS-CoV-2 is believed to be a zoonotic virus from bats ${ }^{12}$ that transmits among humans and infects various organs, including the lungs ${ }^{13}{ }^{\text {brain }},{ }^{14}$ kidneys, ${ }^{15}$ liver, ${ }^{16}$ and heart. ${ }^{17}$ Coronaviruses are positive single-strand ribonucleic acid (RNA) viruses with positive-sense, single-stranded RNA that can be directly accessed by the ribosomes of the host to produce proteins and uses a simple reproduction pathway (viruses with polycistronic mRNA) or a more complex transcription pathway (for which proteolytic processing of polyproteins, ribosomal frameshifting, and subgenomic mRNAs may be used). ${ }^{18}$ Antiviral agents are categorized into virucidal (destroy the supercritical membrane 
and the virion protein capsid or penetrate into the virion and destroy the viral genome; irreversible) and virustatic (substances that possess reversible interaction with the virus and parameters like $\mathrm{pH}$, temperature, and dilution can cause the release of virions). ${ }^{19,20}$

The functionalization of quantum and nanomaterials has been used in antiviral research due to their unique chemical, and physical properties in the biomedical field, including quantum dots, metal and metal oxide nanoparticles (NPs). ${ }^{21-26}$ The poor stability of NPs (nanosheets and nanofibers), the cytotoxicity of degradation products (precious metal NPs), and the complexity of synthetic techniques restrict the antiviral application of NPs. ${ }^{23,27-33}$ Hence, the establishment of stable, biodegradable virucidal nanomaterials using a facile approach is urgently needed.

The applications of quantum science and nanoscience in developing new antiviral strategies to prevent viral infection are discussed in this review. Some of the latest broad-spectrum antiviral strategies that might be useful in thwarting SARS-CoV-2 in the near future are presented. Furthermore, the nanoscience and quantum science phenomena in antiviral therapy are comprehensively explored.

\section{Application of nanoscience in antiviral strategies}

In general, the nanoscience field studies the correlation of atomic and molecular structures with the nanometer scale materials phenomena, while the engineering of functional nanomaterials is known as nanotechnology. ${ }^{34,35}$ The quantum and nano-based materials possess amenable physical, chemical, and biological properties that enhance their potential in comparison with bulk materials. ${ }^{34,36}$ There is great potential for using quantum and nanomaterials against viral infections such as SARS-CoV-2. We categorized the quantum and nanomaterials into three groups based on their potential to prevent the dissemination of the viral infection, including preventive application, diagnostic application, and therapeutic application. Nanocarriers or functionalized NPs can deliver drugs to the target cells and prevent virus replication and infection. The incorporation of nanomaterials into PPE and coating of surfaces can prevent the attachment of viruses or destroy virions. Quantum and nanomaterials in sensors can give sensitive, accurate, and rapid detection of virus infection rates in the primary phase. Quantum and nano-based materials in vaccines can help in the prophylaxis or treatment of COVID-19. Nanomaterials can terminate the lifecycle of SARS-CoV-2 in a different phase. Nanomaterials can inhibit virus binding to the target cells, prevent cell penetration by blocking the angiotensinconverting enzyme 2 (ACE2) receptor, prevent internalization of the virion to the cell and trafficking, prevent escaping from the endosome, inhibit membrane fusion and uncoating, and stop the release of the viral genome. ${ }^{37,38}$ Nanomaterials can be incorporated into PPE and prevent the entrance of viruses to the target cell. Nanocarriers can be applied to prevent the entrance of viruses to the lung or alveoli by delivery of the drug to the respiratory system. Nanostructured materials prevent the binding of the virus to the host cell by targeting the viral spike glycoprotein or ACE2 receptor and prevent entrance of virions to the target cell. The S protein is a key target for diagnostics, therapeutic antibodies and vaccines. ${ }^{6}$ Nano-based materials can systematically inactivate SARS-CoV-2 by neutralization of the virus or in combination with photodynamic therapy. Furthermore, nanoparticles can boost the immune system by immunomodulation during infection or when used in combination with a vaccine to prevent infection. ${ }^{3,37,39-41}$ Transmembrane serine protease 2 (TMPRSS2) on the surface of the target cell facilitates cellular entry through protease activity. ${ }^{21}$ Nanostructured materials inhibit or reduce the activation/expression of the cellular protease TMPRSS2, which allows the spike proteins, potentially suppressing SARS-CoV-2 infection. ${ }^{26,42}$ Nanostructured materials can inhibit the budding and release of the virus. ${ }^{21}$ Fig. 1 illustrates the life cycle of SARS-CoV-2 and possible targets for nanomaterials.

\section{Diagnostic applications}

The primary phase in the treatment is the detection of the virus and isolation of infected people to avoid the spread of viral infection. ${ }^{43}$ The surface modification of quantum and nanomaterials in diagnostic applications is based on paper-based biomolecular sensors using aptamers, beacons, and nucleic acids, a plasmonic-based colorimetric assay using RNA/DNA, thermoplastic chips using oligos, and antigen-binding and antibody capture assays using antibodies. ${ }^{44-50}$ Table 1 shows the diagnostic applications of quantum and nanomaterials in viral detection. Nucleic acid amplification and immunoassays employing PCR are reliable techniques for the detection of viral nucleic acids and proteins, respectively. The precision of the results depends on the sensitivity of the materials and instruments. The immune colloidal gold method is an immunoassay approach in which antibodies are labeled with plasmonic gold nanoparticles, which enable the fluorescent readout after the capturing of the antigen by the antibody. Using gold NPs enhances near-infrared fluorescence by up to 100 times. Nanowire field effect transistors with immune colloidal gold used for direct electrical detection and real-time detection of virions rely on conductance alteration after antibody-antigen binging. Furthermore, graphene quantum dots and gold embedded polyaniline nanostructures ameliorate the detection of the virus in the virus accumulation step. ${ }^{51}$ The limitations of professional medical staff and instruments necessitate artificial intelligence (AI) and the development of portable diagnosis tools to help clinical diagnosis, guarantee the accuracy of results and enhance the detection process. ${ }^{51}$

\section{Preventive application: disinfectant}

After detection of the virus, the next approach is stopping transmission of infection using a disinfectant and personal protective equipment (PPE), such as facemasks, gloves, goggles, and respirators. Incorporation of quantum and nanomaterials into the matrix of the polymer of materials can prevent the 


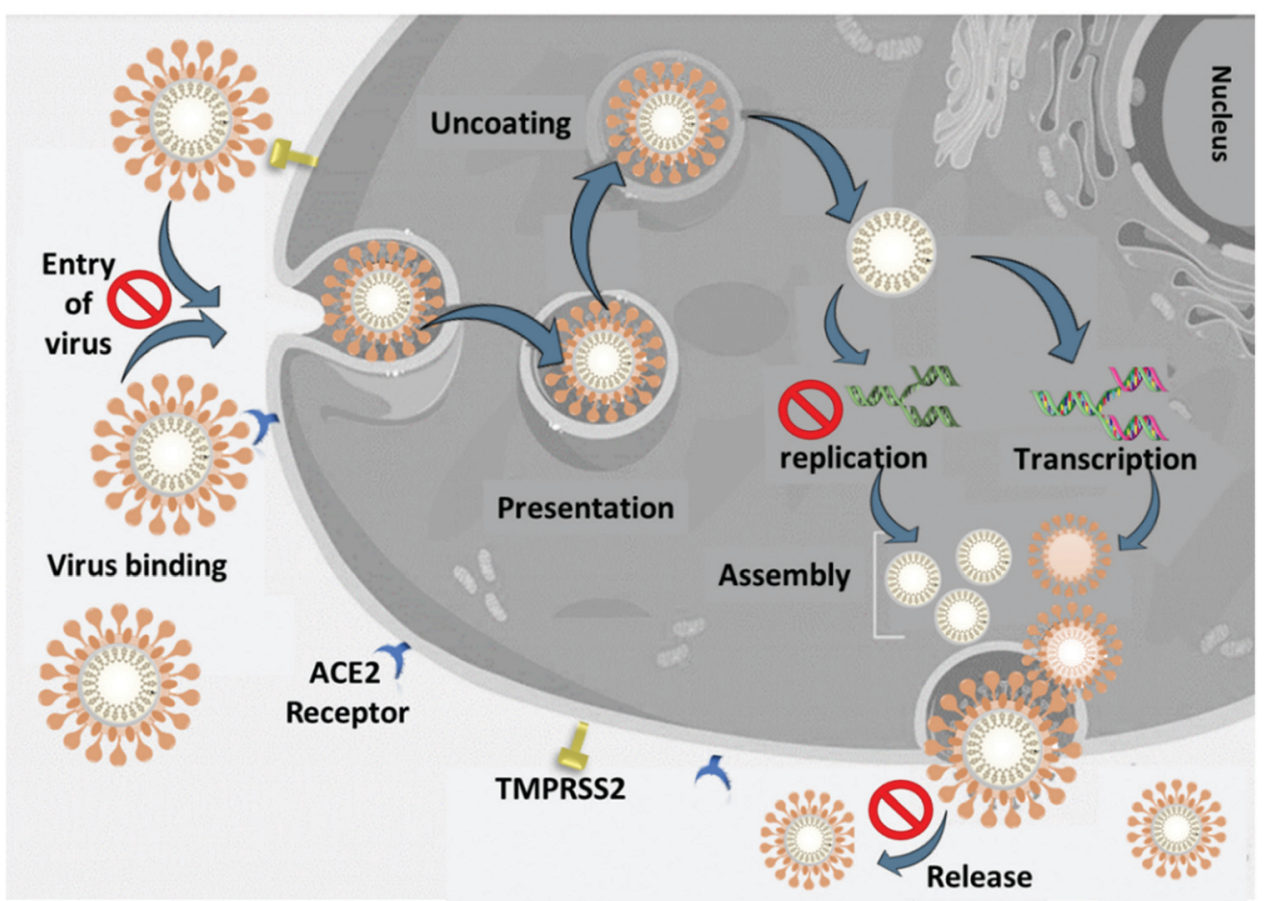

Fig. 1 The life cycle of SARS-CoV-2 and possible targets for nanomaterials. SARS-CoV-2 binds to an ACE-2 receptor on the surface of the target cell. Nanomaterials can prevent binding to ACE-2 and inhibit entry of the virus to the cell. TMPRSS2 through protease activity facilitates cellular entry and reduces or inhibits expression/activation of the cellular protease. The virion is internalized into the endosome and uncoated due to the low $\mathrm{pH}$ of the endosome, then the viral genome is released for protein synthesis. Nanomaterials inhibit replication of the viral genome. The new infectious virions are assembled and released. The nanoparticles can inhibit the release of the virus.

dissemination of the virus by prevention of the lifecycle of SARS-CoV-2, ROS generation, release of toxic chemicals to the virus, surface oxidation, and degradation of nanoparticles. ${ }^{64}$
The unique physical and chemical properties and the high surface area-to-volume ratio of nanomaterials can be applied to absorb or eliminate SARS-CoV-2. The incorporation of

Table 1 Applications of quantum and nanomaterials in viral detection

\begin{tabular}{llll}
\hline Nanoparticles & Purpose & Virus & Mechanism of antiviral action \\
\hline Chiral Au NPs-quantum dot & Viral detection & Coronavirus and Chiral plasmon-exciton system
\end{tabular}

nanocomposite

Quantum dots

Magnetic-derivatized plasmonic molybdenum trioxide quantum dots (MP-MoO 3 QDs)

Viral detection Viral detection fowl adenovirus, avian influenza A (H4N6) virus RSV H1N1 H3N2
Streptavidin interacts with G-protein in virus A core-satellite immunocomplex is formed 53 between the antibody-conjugated nanomaterials (Ab-MP- $-\mathrm{MoO}_{3}$ QDs and Ab-gCNQDs) and their interaction resulted in the modulation and gradual enhancement of the fluorescence intensity of the detection

Europium-doped fluorescent Si NPs Fluorescence-based sandwich immunoassay detection of virus Specific and rapid viral detection H5N2

$\mathrm{Fe}_{3} \mathrm{O}_{4}$ NPs

Unmodified Au NPs

$\mathrm{Au}$ NPs

Au NPs

Silica-shelled magnetic nanobeads (Mag NBs) and Au NPs $\mathrm{Au} /$ iron oxide magnetic NP-decorated CNTs Poly(DL-lactide-co-glycolide)encapsulated superparamagnetic iron oxide NPs Graphene-Au NPs nanohybrid Colorimetric-based viral detection H3N2 assay Viral detection

Foot-and-mouth Peroxidase-mimic enzymatic reaction

Colorimetric immunosensor viral H1N1 detection

Ultra-sensitive colorimetric assay for viral detection

High selectivity and sensitivity detection of viral DNA Improved viral detection

Viral detection via colorimetric immunoassays disease virus

$\mathrm{H} 3 \mathrm{~N} 2, \mathrm{H} 1 \mathrm{~N} 1$ H1N1, norovirus DNA hybridization

H1N1

HIV (HIV-1 p24 Binding to HIV-1 p24 antigen 55

BVDV-RNA-based PNA-induced aggregation of the 57 $\mathrm{Au}$ NPs

Viral surface deposition of NP-mAb

Mag NB-mediated target signal amplification and 60 separation by the enzyme-like activity of Au NPs

Virus isolation and targeting through magnetic extraction

Norovirus-like Intrinsic peroxidase-like activity particles 
graphene foam in reusable masks can damage the microorganism by conduction of an electrical charge. The application of titanium oxide and silver nanoparticles in photocatalytic coatings (light-mediated) destroys the virion cell membrane and can therefore be used for developing a durable and selfsterilizing formula. Cellulose nanofibers and nanostructured materials can be integrated into facemasks to increase their inhibitory effect and make them more breathable with a higher filtration capability. ${ }^{65-67}$ The adverse effect of nanostructured materials on the environment and humans can be inhibited by employing polymeric lipid-based and biodegradable nanomaterials. ${ }^{64}$ The application of nano-based materials in antiviral therapy, especially against SARS-CoV-2, is restricted due to lengthy and strict regulatory affairs. ${ }^{68}$

\section{Preventive application: vaccines}

Vaccines play a critical role in SARS-CoV-2 infection annihilation. The establishment of a vaccine requires the genome and protein structure of the target virus. Various types of vaccine are in use, including nucleic acid-based vaccines, live-attenuated vaccines, inactivated vaccines, subunit vaccines, nanoparticle-based vaccines, and peptide-based vaccines. ${ }^{69}$ Nanoparticle-based vaccines are appropriate for the delivery of antigens and as mimics of the viral structure or as adjuvants. Cationic liposomes and polymeric nanoparticles are applied in DNA vaccines as a nanocarrier to cross the cell membrane. ${ }^{70}$ Nanoparticle-based vaccine designs include: (1) virus-like proteins (antigen encapsidation and antigen expression); and (2) synthetic nanoparticle platforms including RNA, DNA, and subunit vaccines. ${ }^{37}$ Table 2 shows the applications of nanoparticles in vaccines.

Metal nanoparticles such as gold NPs can be used as virucidal agents to enhance mucosal cellular immunity and are used in vaccines and colorimetric-based viral detection assays. Silver NPs have virucidal properties and prevent viruses from entering the host cell. Iron oxide NPs are applied for rapid viral removal and rapid viral detection, possess antiviral properties, and are used in a vaccine against SARS-CoV-2. ${ }^{25,26}$ Silica NPs are employed as vaccine adjuvants and in immunization to prevent viral entry to the target cell. Silica can also be used in fluorescence-based sandwich immunoassay detection. Among carbon-based NPs, fullerenes have virucidal features and prevent viral binding and viral entry to cells. Single-wall carbon nanotubes possess immunization and viral inhibition properties and graphene has only antiviral potency. Quantum dots have antiviral activity and are used for viral detection while carbon dots can be used for viral inhibition. Inorganic nano-based materials such as nanomicelles and niosomes are used for drug delivery with antiviral efficacy. Polymer nanoparticles act as antiviral agents and are employed in vaccine immunization and efficient antiviral delivery. Dendrimers with antiviral activity inhibit virus encapsidation in the mRNA vaccine. ${ }^{11}$

\section{Therapeutic applications}

The surface modification and functionalization of quantum and nanomaterials in targeted drug delivery and vaccination play a critical role in terminating the viral infection life cycle. Nanocarriers such as micelles, liposomes, dendrimers, and nanocapsules can stop virus replication as a drug carrier by entrapping, encapsulating, dissolving, absorbing, or chemically bonding to the virus and causing it to malfunction. Inorganic drug carriers are metal and metal oxide nanoparticles including titanium oxide, zinc oxide, iron oxide, gold, copper, silver, and zinc. Organic nanocarriers such as liposomes, niosomes, polymeric nanoparticles, nanocapsules, micelles, and dendrimers are used in drug delivery. Table 3 presents the therapeutic application of quantum and nanomaterials. ${ }^{37,68,77-80}$ Nanodelivery of biological agents needs intelligent nanocarrier designs embracing approaches similar to the prodrug method. A rational development and clinical translation of nanomedicine will require several strategic track developments (fast paced and slower paced ones) tackling the right translational challenge and fostering more nanomedicine research. ${ }^{40}$ Strategic guidelines proposed

Table 2 List of nanomaterials used in vaccination to eradicate viral infection

\begin{tabular}{|c|c|c|c|c|c|c|}
\hline Nanoparticles & Purpose & Virus & Model organism & $\begin{array}{l}\text { Biomolecule loaded/ } \\
\text { conjugated }\end{array}$ & Mechanism of antiviral action & Ref. \\
\hline Iron oxide NPs & $\begin{array}{l}\text { Antiviral agents } \\
\text { and a vaccine }\end{array}$ & SARS-CoV-2 & & $\begin{array}{l}\text { Spike protein receptor } \\
\text { binding domain (S1-RBD) }\end{array}$ & $\begin{array}{l}\text { Conformational changes to viral } \\
\text { proteins, viral inactivation }\end{array}$ & 71 \\
\hline Modified dendrimer NPs & mRNA vaccine & Zika virus & C57BL/6 mice & $\begin{array}{l}\text { Venezuelan equine } \\
\text { encephalitis virus (VEEV) } \\
\text { replicon RNAs }\end{array}$ & $\begin{array}{l}\text { Activation of both viral E } \\
\text { protein-specific IgG responses } \\
\text { and } \mathrm{CD} 8^{+} \mathrm{T} \text {-cell }\end{array}$ & 72 \\
\hline Dendrimer NPs & mRNA vaccine & $\begin{array}{l}\text { H1N1, } \\
\text { Ebola virus }\end{array}$ & $\begin{array}{l}\text { BALB } / c \text { mice and } \\
\text { wild-type female } \\
\text { C57BL } / 6\end{array}$ & $\begin{array}{l}\text { mRNA replicons (multiple } \\
\text { antigen-expressing } \\
\text { replicons) }\end{array}$ & $\begin{array}{l}\text { Activation of both antibody } \\
\text { responses and CD8 } 8^{+} \text {T-cell }\end{array}$ & 73 \\
\hline $\begin{array}{l}\text { Double-layered polypeptide } \\
\text { (nucleoprotein epitopes at core } \\
\text { coated with matrix protein } 2 \\
\text { ectodomain epitopes) NPs }\end{array}$ & NPs vaccine & $\begin{array}{l}\text { Influenza } \\
\text { A virus }\end{array}$ & $\mathrm{BALB} / c$ strain & & $\begin{array}{l}\mathrm{CD}^{+} \mathrm{T} \text { cells involved in } \\
\text { protection against virus }\end{array}$ & 74 \\
\hline $\begin{array}{l}\text { Ammonium-functionalized } \\
\text { single-walled carbon nanotubes } \\
\text { (SWCNTs) }\end{array}$ & Vaccine & GCRV & Grass carp & pEGFP- $v p 5$ vaccine & $\begin{array}{l}\text { Activation of pro-inflammatory } \\
\text { factors (IL-1 } \beta, \text { TNF- } \alpha \text { ) and DNA } \\
\text { vaccine-mediated immunity } \\
\text { against virus }\end{array}$ & 75 \\
\hline Functionalized SWCNTs & Vaccine & GCRV & Grass carp & $\begin{array}{l}\text { Recombinant VP7 subunit } \\
\text { vaccine }\end{array}$ & $\begin{array}{l}\text { VP7 subunit vaccine-mediated } \\
\text { immunity against virus }\end{array}$ & 76 \\
\hline
\end{tabular}


Table 3 Nanocarriers in therapeutic applications

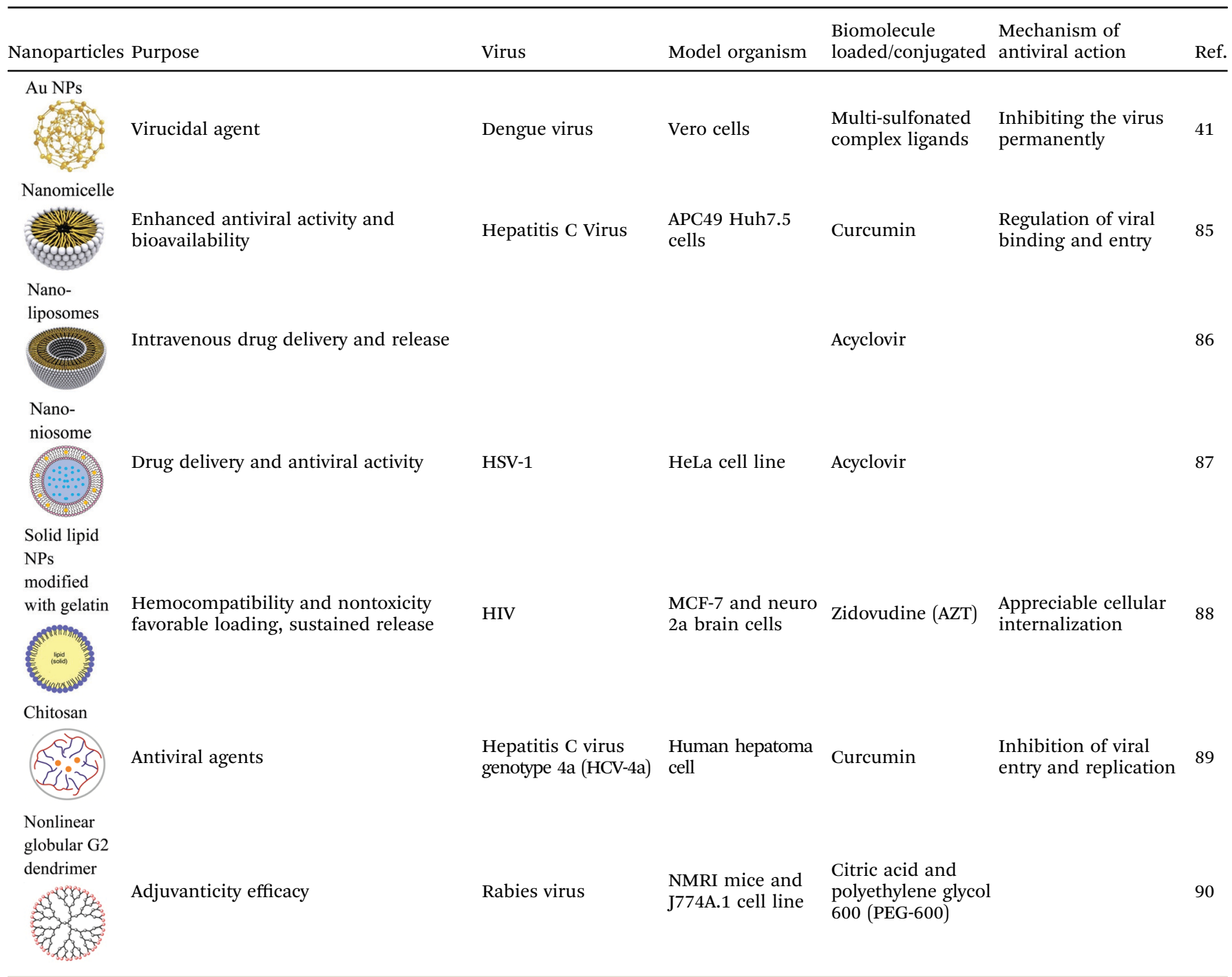

for the advancement of antiviral/anticancer nanomedicines are significant, highlighting the right fields and specific tools to develop SARS-CoV-2 nanomedicines. ${ }^{81,82}$ The emergence of novel coronavirus SARS-CoV-2 varients is increasing at an alarming rate. A comprehensive strategy with complete perception and versatility to solve the expanding distress could be accomplished by the employment of nanoscience and nanotechnology. However, in parallel with the resourceful and accurate implementation of nano-based treatments, there is an eventual requirement for a precise understanding of virus infection. Besides, to increase the efficiency of the nano-based methods in the conquest against SARS-CoV-2, a list of advanced antiviral nanosystems is also a prerequisite. Therefore, several nanobased inhibition and diagnosis studies against viruses have been initiated. The multifunctional approach of viral inhibition is based on the form and type of NPs used. Hence, to realize the antiviral activity of nanomaterials, a complete overview of various forms and types of nano-based structures is required. ${ }^{40,83}$ Polymeric nanoparticles are in the range of 10 nanometers to one micron that includes colloidal nanosized particles fabricated from biocompatible and biodegradable, synthetic, or natural polymers. Polymeric nanoparticles can encapsulate therapeutic or diagnostic materials within the polymer matrix and inhibit enzymatic degradation and enhance their permeability, stability, toxicity, biocompatibility, biodegradability, transport efficacy, and cargo bioavailability by control/ release of vaccine targets or by being chemically conjugated on the surface for targeted delivery and diagnostic applications. ${ }^{84}$

Based on the type of nanoformulation, particles will have a positive or negative charge on their surface. Nanoemulsions, carbon nanotubes, cyclodextrin inclusion complexes, polymeric nanoparticles, silver NPs, nanosuspensions, and solid lipid nanoparticles with a positive charge have high penetration rates into the negatively charged surface, high uptake, high loading capacity, high bactericidal activity, high cellular uptake, and enhance immune response. Micelle, liposome, and dendrimer with negative charges possess high entrapment efficiency, do not interact with the biological environment, do not show toxicity. The weakly negative charge is preferred as it undergoes lesser uptake by phagocytes. ${ }^{24}$ 


\section{Applications of quantum science in antiviral strategies}

Quantum dots are zero-dimensional nanomaterials in the range of 1 to 10 nanometers, including graphene quantum dots, polymer dots, carbon quantum dots, zirconium QDs, and fullerenes. They are an extremely attractive platform in nanomedicine due to their low toxicity. ${ }^{21}$ Quantum dots possess tunable optical wavelengths while nanoparticle possesses tunable plasmonic properties, which makes quantum dots suitable for conjugation with a highly fluorescent probe for viral detection and continuing fluorescent molecular imaging applications. The importance of quantum dots in antiviral properties is based on a biocompatible carrier for clinical approaches and interdisciplinary study. Quantum dots can be used in antiviral therapy, biosensing, biolabeling, drug delivery, and photodynamic therapy for SARS-CoV-2. The main role of quantum dots is in labeling and as carriers of antiviral therapeutics. The life cycle of the virus can be blocked in different phases, including inhibiting binding of the virus to the target cell, preventing virus translation and eIF4G cleavage, reducing phosphorylated p38 kinase expression, suppressing ROS generation by free radical activity, preventing virus-induced PGE2 production, and inducing downregulation of pro-viral proteins or upregulation of intracellular antiviral proteins. ${ }^{91-94}$ The sensitivity of zirconium QDs for detection of avian coronavirus is better than that of conventional enzyme-linked immunoassay (ELISA) techniques. The optical properties of QDs could help in the diagnosis of COVID-19 and QDs could play an effective role as imaging probes. ${ }^{21}$

\section{Quantum and nanoparticle phenomena in antiviral therapy}

The antiviral potency of quantum and nanomaterials depends on their size and shape, the structure of the molecular ions on the surface of the particles, and the equilibrium distance. Quantum dots interact with the virus via van der Waals forces, which cause thermal, electrodynamic, and medium fluctuation, which leads to dispersion forces. ${ }^{95,96}$ The next interaction is based on the local field enhancement effect on the virus receptor that inhibits cell entrance by weakening the chemical bonds or suppressing the active center in the virus, which causes cell destruction or changes the molecular groups on the receptor. Furthermore, the occurrence of the resonance is related to local field enhancement and, very probably, to the occurrence of the "collective" mode leading to a strong narrow absorption line in J-aggregate. ${ }^{91-94,106}$ There is an electric field fluctuation in this physical system, then the induced dipole moment induces the following electric field. The vacuum field fluctuation between the virus and the nanoparticles inhibits the infectious activity of the virus. ${ }^{95}$ The mechanism of action for all types of DNA and RNA viruses, enveloped and nonenveloped viruses, is similar for all types of nanoparticles. The quantum and nanoparticles cause alterations to the receptor geometry and prevent the binding of the virus to the target cell. $^{73-75}$

Lipid envelope antiviral disruption (LEAD) is the most recently introduced strategy to selectively destroy the envelope of a virus (e.g., SARS-COV-2, Zika, Ebola, HIV, influenza, measles virus, herpes viruses), and alter amyloid fibrils, which cause viral infection, and results in the loss of virus particle infectivity, and thus can be used for the development of broad-spectrum antiviral therapy. ${ }^{97-99}$ Fig. 2 illustrates the broad-spectrum antivirals targeting viral membranes. ${ }^{100}$ There are different types of antiviral agents that impact on the lipid membrane properties of enveloped viruses, including molecular tweezers, photosensitizers, rigid amphipathic fusion inhibitors, and amphipathic peptides. ${ }^{97,98,100}$ The photosensitizer mechanism is based on oxidizing unsaturated phospholipids. The two proposed mechanisms of action of amphipathic antiviral peptidesis (AVPs) are based on (i) permeabilizing lipid membranes with high partition coefficient and destabilizing lipid membranes independent of membrane curvature, and (ii) lysing of the cell membrane after the critical density of pores is formed, and selectively forming pores in the highly curved membrane. The rigid amphipathic fusion inhibitors prevent virus-host cell fusion by the positive curvature effect and can also oxidize unsaturated phospholipids to alter the fluidity of the membrane. ${ }^{97,98,101}$

The shape and size of quantum dots are tunable in the range of 1 to 10 nanometer to effectively target and penetrate SARSCoV-2. ${ }^{94}$ The spike protein on the surface of the coronavirus can be disabled or sequestered by the positive surface charge of cationic carbon-based QDs. ${ }^{102}$ The cationic surface charge of QDs interacts with the genome of SARS-CoV-2 and causes the generation of ROS within the virion, which prevents negativestrand RNA replication. ${ }^{94,103,104}$ Furthermore, quantum dots activate interferon- $\alpha$ gene production and inhibit viral replication. The cationic charge on the surface of quantum dots causes virus aggregation through electrostatic interaction and decreases viral infection. ${ }^{94,105}$ Applying radiation with an electromagnetic field of a fixed (resonant) frequency to a virusquantum dot complex leads to destroying the nucleocapsid. ${ }^{106}$ However, the precise mechanism of action of quantum dots in antiviral therapy remains unclear. ${ }^{94,107}$ Fig. 3 illustrates the SARS-CoV-2 structure and quantum phenomena in antiviral therapy.

There are three mechanisms for ROS generation by QDs: (i) excitation of the QDs results in an electron-hole pair, either the hole or the electron may be trapped in the surface defect, causing a metastable charge-separated "dark" state with a long lifetime, (ii) a reductive process that transfers an electron to molecular oxygen, leading to ROS, and (iii) an oxidative process where a hole is transferred to an electron donor. However, the number of trap states is determined by the nature of the QDs surface, which in turn impacts charge transfer among the absorbed molecule, the photoexcited electrons, and the holes. Besides, conduction and valence band redox potential determine which molecules can be reduced or oxidized to generate ROS. The position of the band edges and the width 


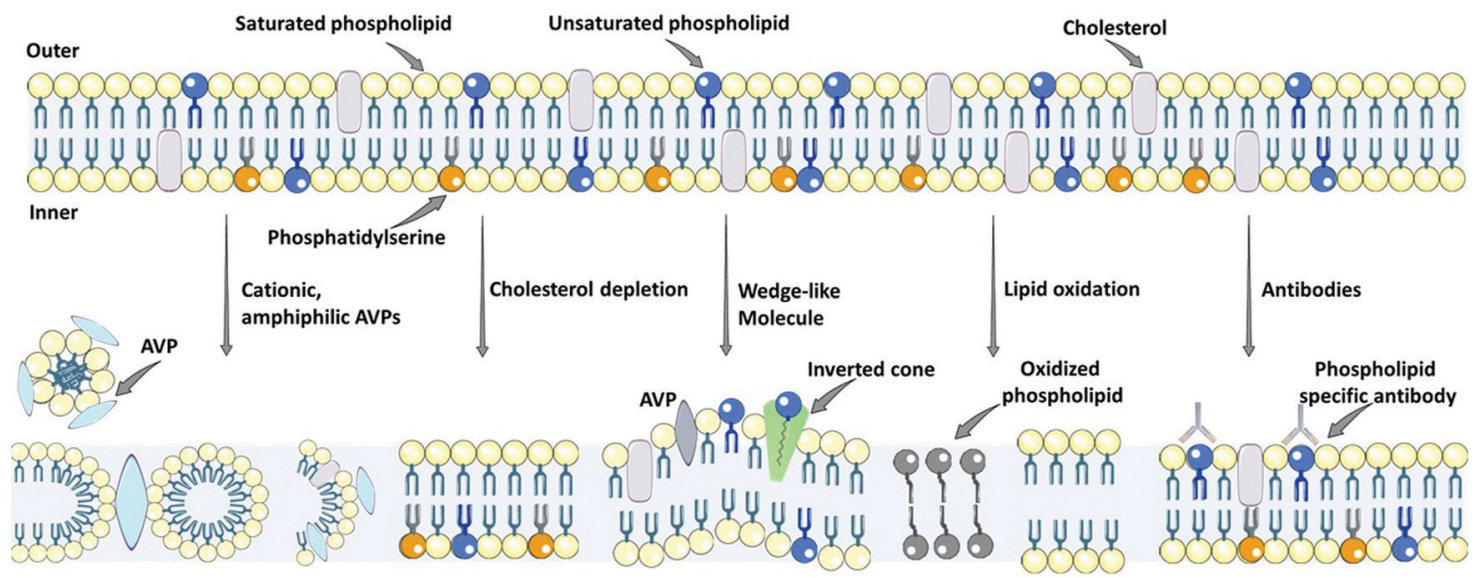

Fig. 2 The removal and addition of lipid species to the lipid composition of the membrane are antiviral strategies that can alter the curvature and fluidity of the membrane. For instance, amphiphilic antiviral peptides (AVPs), some cationic, have detergent-like features at a high amount and can generate pores and cause the micellization of the viral membrane. Polyunsaturated endoplasmic reticulum-targeting liposomes have shown potential as broadspectrum antivirals by depleting cellular and viral membranes of cholesterol; cholesterol depletion decreases the fluidity of the membranes and reduces the negative-curvature transitions that are essential for the fusion between the cellular membrane and the virus. Wedge-like or inverted-cone-shaped molecules and some amphiphilic AVPs can increase the spontaneous positive curvature of the viral membrane lipid bilayer, raising the barrier of energy required to power membrane fusion mediated by viral fusion proteins. Similarly, membrane-targeting type II photosensitizers generate singlet oxygen within the plane of the viral membrane, and this singlet oxygen oxidizes unsaturated phospholipids and induces changes in the nanoarchitecture of the viral membrane that are not conducive to membrane fusion. The clustering of oxidized phospholipids causes differential lipid packing, decreased fluidity, increased positive curvature, increased area per lipid molecule, and reduced membrane thickness. Phospholipid-specific antibodies can target particular phospholipid species that are enriched in some viral membranes (such as phosphatidylserine) and thus block viral attachment and entry.

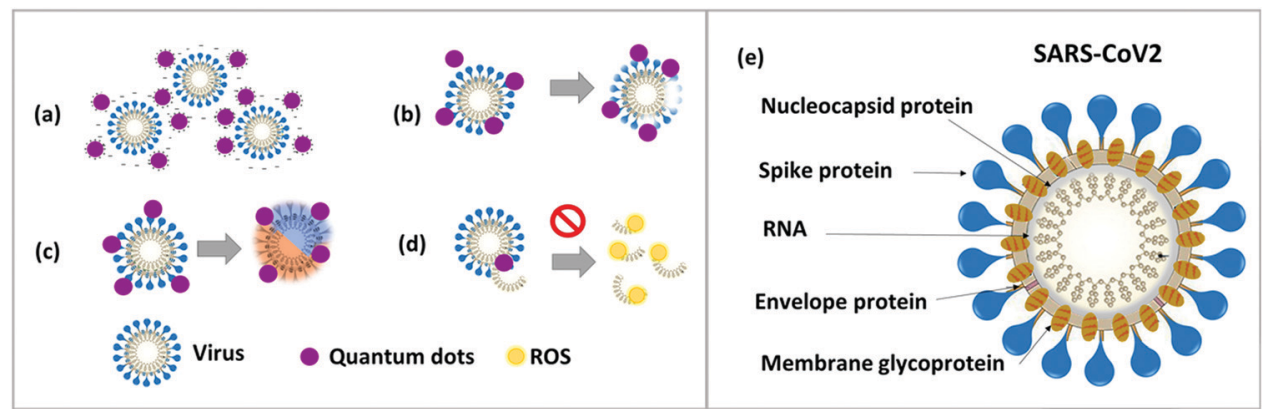

Fig. 3 The structure of SARS-CoV-2 and the quantum and nanoparticles mechanism of action in antiviral therapy. (a) Aggregation of viruses due to electrostatic interaction, (b) the local field effect causes alteration in receptors and prevents entry of the virus to the target cell, (c) the physical interaction (van der Waals forces) of the virus and quantum dots leads to thermal and electrodynamic fluctuations that initiate dispersion forces, (d) generation of ROS from quantum and nanostructure materials causes activation of interferon- $\alpha$ gene production and prevents genome replication, and (e) the structure of SARS-CoV-2 including nucleocapsid protein, membrane glycoprotein, spike protein, envelope protein, and RNA.

of the bandgap are dictated by QD materials, size, and the solvent. ${ }^{108}$

In 2019 Huang et al. showed that benzoxazine monomerderived carbon quantum dots (BZM-CQDs) possess infectionblocking potential against non-envelope viruses (adenovirusassociated viruses and parvoviruses) and flaviviruses. Their in vitro studies confirmed that BZM-CQDs directly binds to the surface of the virus and prevents virion functionality in the first step of virus activity. ${ }^{29}$ The viral protein expression and viral titers assays confirmed that CQDs at a noncytotoxic concentration possess significant antiviral activity against porcine reproductive and respiratory syndrome (PRRSV) and pseudorabies virus (PRV) as model RNA and DNA viruses, respectively. Mechanism study demonstrated that CQDs activate interferon- $\alpha$ production and the expression of interferon-stimulated genes, which suppress replication and cause viral inhibition. ${ }^{105,109}$ The interaction of functionalized CQDs groups with HCoV-229E protein-S receptors prevents virus entry, inhibits viral replication and inhibits host cell infection. This could possibly be described by an interaction between the CQDs and a cell surface protein causing signal transduction, disturbing replication of the virus, or by interaction with cytosolic proteins as CQDs are internalized. ${ }^{23}$ The functionalized CQDs are extremely encouraging for replacing currently used antiviral agents such as IFN and ribavirin, which are known to have major side effects such as short-term memory loss, extrapyramidal effects, deficits in executive functions, and confusion. ${ }^{23}$ 


\section{Conclusion and future perspective}

The most pandemic-causing viruses are membrane-enveloped viruses and hence targeting viral membranes (viz. enveloped virus disruption) would be advantageous to further understand based on nano and quantum concepts. There are great prospects for impactful research on more effective antiviral agents using quantum science and nanoscience-enabled nanomaterials in vaccines, medical equipment, and consumables, rapidly manufacturing lab-on-a-chip detective nano-sensors, and creating anti-infective surface nano-coatings. The remarkable quantum dots could easily target viruses to prevent entry and replication. Recently, the most important topic in the medical field is surface detection of virus that rapidly responds to the infection or disease. In this review article, nano and quantum materials are proposed to overcome antiviral therapy challenges. From the present investigations, quantum and nanomaterials possess ample applications in detection, prevention, and treatment, which can eliminate SARS-CoV-2. The introduced LEAD strategy establishes a conceptual framework to advance antiviral agents that selectively target virion membranes and prevent infection by enveloped viruses. This review explored the quantum and nano-based phenomena in antiviral therapy. The van der Waals forces between particles and viruses can cause electrodynamic and thermal fluctuations that initiate dispersion forces. Furthermore, the electrostatic charges of quantum and nanoparticles lead to aggregation of the virus and prevent its entrance to the target cell. The local field enhancement effect of quantum and nanoparticles causes a change in the receptors and terminates the virus function. The ROS generation of quantum and nanoparticles inhibits genome replication by the virus. The optimized functionalization of quantum and nanoparticles can be efficiently applied in antiviral therapy and overwhelm the global health threats such as SARS-CoV-2 and other viral infections in coming years. From our point of view, quantum and nanomaterials can be applied in quantum and nano-based antiviral strategies by preventing binding and entrance of the virus to the host cell, trafficking, escaping from the endosome, membrane fusion, and uncoating and viral genome replication. The phospholipid membrane comprising the viral envelope is an opportune target, especially if nanoscience-based strategies can be further optimized to selectively target the viral membrane without impacting other membranes, such as human cell membranes. It will be necessary to extend the analysis and characterize the pharmacokinetic properties of LEAD antiviral agents in order to understand how long they stay in the body and at what concentrations they can be used in the body. Besides, it is critical to assess the toxicokinetic properties of LEAD antiviral agents in animal models for assessing treatment safety and efficacy as part of the next stage of preclinical testing. Quantum and nanomaterials can be used in diagnostic kits, vaccines, targeted drug delivery, and antiviral treatment. Further studies are required to discover the long-term toxicity and side effects of quantum and nanomaterials in antiviral therapy.

\section{Funding}

The authors received no specific funding for this work.

\section{Conflicts of interest}

The authors declare no competing financial interest.

\section{References}

1 J. A. Jackman, B. K. Yoon, L. Ouyang, N. Wang, A. R. Ferhan, J. Kim, T. Majima and N. Cho, Biomimetic Nanomaterial Strategies for Virus Targeting: Antiviral Therapies and Vaccines, Adv Funct Mater, 2020, 2008352.

2 H. V. Fineberg, Pandemic Preparedness and Response Lessons from the H1N1 Influenza of 2009, N. Engl. J. Med., 2014, 370, 1335-1342.

3 N. J. Cho and J. S. Glenn, Materials science approaches in the development of broad-spectrum antiviral therapies, Nat. Mater., 2020, 19, 813-816.

4 S. Jiang and Z. L. Shi, The First Disease X is Caused by a Highly Transmissible Acute Respiratory Syndrome Coronavirus, Virol. Sin., 2020, 35, 263-265.

5 R. Medhi, P. Srinoi, N. Ngo, H.-V. Tran and T. R. Lee, Nanoparticle-Based Strategies to Combat COVID-19, ACS Appl. Nano Mater., 2020, 3, 8557-8580.

6 D. Wrapp, N. Wang, K. S. Corbett, J. A. Goldsmith, C.-L. Hsieh, O. Abiona, B. S. Graham and J. S. McLellan, Cryo-EM structure of the 2019-nCoV spike in the prefusion conformation, Science, 2020, 367, 1260-1263.

7 L. Zhang, D. Lin, X. Sun, U. Curth, C. Drosten, L. Sauerhering, S. Becker, K. Rox and R. Hilgenfeld, Crystal structure of SARS-CoV-2 main protease provides a basis for design of improved $\alpha$-ketoamide inhibitors, Science, 2020, 368, 409-412.

8 Education in a post-COVID world: Nine ideas for public action International Commission on the Futures of Education. 2020.

9 J. J. V. Bavel, K. Baicker, P. S. Boggio, V. Capraro, A. Cichocka, M. Cikara, M. J. Crockett, A. J. Crum, K. M. Douglas and J. N. Druckman, et al., Using social and behavioural science to support COVID-19 pandemic response, Nat. Hum. Behav., 2020, 4, 460-471.

10 R. N. Rimal and M. K. Lapinski, Why health communication is important in public health, Bull. W. H. O., 2009, 87, 247.

11 J. K. Patra, G. Das, L. F. Fraceto, E. V. R. Campos, M. D. P. Rodriguez-Torres, L. S. Acosta-Torres, L. A. DiazTorres, R. Grillo, M. K. Swamy and S. Sharma, et al., Nano Based Drug Delivery Systems: Recent Developments and Future Prospects, J. Nanobiotechnol., 2018, 16, 71.

12 K. G. Andersen, A. Rambaut, W. I. Lipkin, E. C. Holmes and R. F. Garry, The proximal origin of SARS-CoV-2, Nat. Med., 2020, 26, 450-452.

13 M. Ackermann, S. E. Verleden, M. Kuehnel, A. Haverich, T. Welte, F. Laenger, A. Vanstapel, C. Werlein, H. Stark and 
A. Tzankov, et al., Pulmonary Vascular Endothelialitis, Thrombosis, and Angiogenesis in Covid-19, N. Engl. J. Med., 2020, 383, 120-128.

14 S. Kremer, F. Lersy, J. De Sèze, J. C. Ferré, A. Maamar, B. Carsin-Nicol, O. Collange, F. Bonneville, G. Adam and G. B. Martin, et al., Brain MRI findings in severe COVID-19: A retrospective observational study, Radiology, 2020, 297, E242-E251.

15 Y. Cheng, R. Luo, K. Wang, M. Zhang, Z. Wang, L. Dong, J. Li, Y. Yao, S. Ge and G. Xu, Kidney disease is associated with in-hospital death of patients with COVID-19, Kidney Int., 2020, 97, 829-838.

16 C. Zhang, L. Shi and F. S. Wang, Liver injury in COVID-19: management and challenges, Lancet Gastroenterol. Hepatol., 2020, 5, 428-430.

17 Y. Y. Zheng, Y. T. Ma, J. Y. Zhang and X. Xie, COVID-19 and the cardiovascular system, Nat. Rev. Cardiol., 2020, 17, 259-260.

18 K. Nakagawa, K. G. Lokugamage and S. Makino, Viral and Cellular mRNA Translation in Coronavirus-Infected Cells, Adv. Virus Res., 2016, 96, 165-192.

19 A. S. Galabov, Virucidal Agents in the Eve of Manorapid Synergy, GMS Krankenhhyg Interdiszip, 2007, 2, Doc18.

20 S. T. Jones, How materials can beat a virus, J. Mater. Sci., 2020, 55, 9148-9151.

21 C. Weiss, M. Carriere, L. Fusco, L. Fusco, I. Capua, J. A. Regla-Nava, M. Pasquali, M. Pasquali, M. Pasquali and J. A. Scott, et al., Toward Nanotechnology-Enabled Approaches against the COVID-19 Pandemic, ACS Nano, 2020, 14, 6383-6406.

22 M. Rai, S. Bonde, A. Yadav, Y. Plekhanova, A. Reshetilov, I. Gupta, P. Golińska, R. Pandit and A. P. Ingle, Nanotechnology-based promising strategies for the management of COVID-19: current development and constraints, Expert Rev. Anti-Infect. Ther., 2020, 1-10.

23 A. Łoczechin, K. Séron, A. Barras, E. Giovanelli, S. Belouzard, Y. T. Chen, N. Metzler-Nolte, R. Boukherroub, J. Dubuisson and S. Szunerits, Functional Carbon Quantum Dots as Medical Countermeasures to Human Coronavirus, ACS Appl. Mater. Interfaces, 2019, 11, 42964-42974.

24 M. Chakravarty and A. Vora, Nanotechnology-based antiviral therapeutics, Drug Delivery Transl. Res., 2020, 3, 1-40.

25 R. Medhi, P. Srinoi, N. Ngo, H. V. Tran and T. R. Lee, Nanoparticle-Based Strategies to Combat COVID-19, ACS Appl. Nano Mater., 2020, 3, 8557-8580.

26 S. Gurunathan, M. Qasim, Y. Choi, J. T. Do, C. Park, K. Hong, J.-H. H. Kim and H. Song, Antiviral Potential of Nanoparticles-Can Nanoparticles Fight Against Coronaviruses?, Nanomaterials, 2020, 10, 1-29.

27 A. Riegger, C. Chen, O. Zirafi, N. Daiss, D. Mukherji, K. Walter, Y. Tokura, B. Stöckle, K. Kremer and F. Kirchhoff, et al., Synthesis of Peptide-functionalized Poly(bis-sulfone) Copolymers Regulating HIV-1 Entry and Cancer Stem Cell Migration, ACS Macro Lett., 2017, 6, 241-246.
28 H. Liu, Y. Bai, Y. Zhou, C. Feng, L. Liu, L. Fang, J. Liang and S. Xiao, Blue and Cyan Fluorescent Carbon Dots: One-pot Synthesis, Selective Cell Imaging and their Antiviral Activity, $R S C$ Adv., 2017, 7, 28016-28023.

29 S. Huang, J. Gu, J. Ye, B. Fang, S. Wan, C. Wang, U. Ashraf, Q. Li, X. Wang and L. Shao, et al., Benzoxazine monomer derived carbon dots as a broad-spectrum agent to block viral infectivity, J. Colloid Interface Sci., 2019, 542, 198-206.

30 B. Villeret, A. Dieu, M. Straube, B. Solhonne, P. Miklavc, S. Hamadi, R. Le Borgne, A. Mailleux, X. Norel and J. Aerts, et al., Silver Nanoparticles Impair Retinoic Acid-Inducible Gene I-Mediated Mitochondrial Antiviral Immunity by Blocking the Autophagic Flux in Lung Epithelial Cells, ACS Nano, 2018, 12, 1188-1202.

31 D. Lauster, M. Glanz, M. Bardua, K. Ludwig, M. Hellmund, U. Hoffmann, A. Hamann, C. Böttcher, R. Haag and C. P. R. Hackenberger, et al., Multivalent Peptide-Nanoparticle Conjugates for Influenza-Virus Inhibition, Angew. Chem., Int. Ed., 2017, 56, 5931-5936.

32 I. Donskyi, M. Drüke, K. Silberreis, D. Lauster, K. Ludwig, C. Kühne, W. Unger, C. Böttcher, A. Herrmann and J. Dernedde, et al., Interactions of Fullerene-Polyglycerol Sulfates at Viral and Cellular Interfaces, Small, 2018, 14, 1800189.

33 Y. Zhou, X. Jiang, T. Tong, L. Fang, Y. Wu, J. Liang and S. Xiao, High Antiviral Activity of Mercaptoethane Sulfonate Functionalized Te/BSA Nanostars against Arterivirus and Coronavirus, RSC Adv., 2020, 10, 14161-14169.

34 S. Bayda, M. Adeel, T. Tuccinardi, M. Cordani and F. Rizzolio, The history of nanoscience and nanotechnology: From chemical-physical applications to nanomedicine, Molecules, 2020, 25, 112.

35 V. S. Sivasankarapillai, A. M. Pillai, A. Rahdar, A. P. Sobha, S. S. Das, A. C. Mitropoulos, M. H. Mokarrar and G. Z. Kyzas, On Facing the SARS-CoV-2 (COVID-19) with Combination of Nanomaterials and Medicine: Possible Strategies and First Challenges, Nanomaterials, 2020, 10, 852 .

36 J. Jeevanandam, A. Barhoum, Y. S. Chan, A. Dufresne and M. K. Danquah, Review on nanoparticles and nanostructured materials: History, sources, toxicity and regulations, Beilstein J. Nanotechnol., 2018, 9, 1050-1074.

37 M. D. Shin, S. Shukla, Y. H. Chung, V. Beiss, S. K. Chan, O. A. Ortega-Rivera, D. M. Wirth, A. Chen, M. Sack and J. K. Pokorski, et al., COVID-19 vaccine development and a potential nanomaterial path forward, Nat. Nanotechnol., 2020, 15, 646-655.

38 C. Weiss, M. Carriere, L. Fusco, I. Capua, J. A. Regla-Nava, M. Pasquali, J. A. Scott, F. Vitale, A. Unal and C. Mattevi, et al., Toward Nanotechnology-Enabled Approaches against the COVID-19 Pandemic, ACS Nano, 2020, 7, 28.

39 J. Hasan, Y. Xu, T. Yarlagadda, M. Schuetz and K. Spann, Yarlagadda PKDV: Antiviral and Antibacterial Nanostructured Surfaces with Excellent Mechanical Properties for Hospital Applications, ACS Biomater. Sci. Eng., 2020, 6, 3608-3618. 
40 G. Chauhan, M. J. Madou, S. Kalra, V. Chopra, D. Ghosh and S. O. Martinez-Chapa, Nanotechnology for COVID-19: Therapeutics and Vaccine Research, ACS Nano, 2020, 14, 7760-7782.

41 A. Zacheo, J. Hodek, D. Witt, G. F. Mangiatordi, Q. K. Ong, O. Kocabiyik, N. Depalo, E. Fanizza, V. Laquintana and N. Denora, et al., Multi-sulfonated ligands on gold nanoparticles as virucidal antiviral for Dengue virus, Sci. Rep., 2020, 10, 1-9.

42 M. Hoffmann, H. Kleine-Weber, S. Schroeder, N. Krüger, T. Herrler, S. Erichsen, T. S. Schiergens, G. Herrler, N. H. Wu and A. Nitsche, et al., SARS-CoV-2 Cell Entry Depends on ACE2 and TMPRSS2 and Is Blocked by a Clinically Proven Protease Inhibitor, Cell, 2020, 181, 271-280.

$43 \mathrm{~J}$. Cohen and K. Kupferschmidt, Labs scramble to produce new coronavirus diagnostics, Science, 2020, 367, 727.

44 M. Vaculovicova, P. Michalek, S. Krizkova, M. Macka and V. Adam, Nanotechnology-based analytical approaches for detection of viruses, Anal. Methods, 2017, 9, 2375-2391.

45 A. Mokhtarzadeh, R. Eivazzadeh-Keihan, P. Pashazadeh, M. Hejazi, N. Gharaatifar, M. Hasanzadeh, B. Baradaran and M. de la Guardia, Nanomaterial-based biosensors for detection of pathogenic virus, TrAC, Trends Anal. Chem., 2017, 97, 445-457.

46 P. Teengam, W. Siangproh, A. Tuantranont, T. Vilaivan, O. Chailapakul and C. S. Henry, Multiplex Paper-Based Colorimetric DNA Sensor Using Pyrrolidinyl Peptide Nucleic Acid-Induced AgNPs Aggregation for Detecting MERS-CoV, MTB, and HPV Oligonucleotides, Anal. Chem., 2017, 89, 5428-5435.

47 G. Seo, G. Lee, M. J. Kim, S. H. Baek, M. Choi, K. B. Ku, C. S. Lee, S. Jun, D. Park and H. G. Kim, et al., Rapid Detection of COVID-19 Causative Virus (SARS-CoV-2) in Human Nasopharyngeal Swab Specimens Using FieldEffect Transistor-Based Biosensor, ACS Nano, 2020, 14, 5135-5142.

48 G. Qiu, Z. Gai, Y. Tao, J. Schmitt, G. A. Kullak-Ublick and J. Wang, Dual-Functional Plasmonic Photothermal Biosensors for Highly Accurate Severe Acute Respiratory Syndrome Coronavirus 2 Detection, ACS Nano, 2020, 14, 5268-5277.

49 B. Udugama, P. Kadhiresan, H. N. Kozlowski, A. Malekjahani, M. Osborne, V. Y. C. Li, H. Chen, S. Mubareka, J. B. Gubbay and W. C. W. Chan, Diagnosing COVID-19: The Disease and Tools for Detection, ACS Nano, 2020, 14, 3822-3835.

50 P. Moitra, M. Alafeef, M. Alafeef, M. Alafeef, K. Dighe, M. B. Frieman, D. Pan, D. Pan and D. Pan, Selective NakedEye Detection of SARS-CoV-2 Mediated by N Gene Targeted Antisense Oligonucleotide Capped Plasmonic Nanoparticles, ACS Nano, 2020, 14, 7617-7627.

51 Z. Tang, N. Kong, X. Zhang, Y. Liu, P. Hu, S. Mou, P. Liljeström, J. Shi, W. Tan and J. S. Kim, et al., A materials-science perspective on tackling COVID-19, Nat. Rev. Mater., 2020, 5, 847-860.
52 S. R. Ahmed, É. Nagy and S. Neethirajan, Self-assembled star-shaped chiroplasmonic gold nanoparticles for an ultrasensitive chiro-immunosensor for viruses, $R S C A d v$, 2017, 7, 40849-40857.

53 E. L. Bentzen, F. House, T. J. Utley, J. E. Crowe and D. W. Wright, Progression of respiratory syncytial virus infection monitored by fluorescent quantum dot probes, Nano Lett., 2005, 5, 591-595.

54 O. J. Achadu, K. Takemura, I. M. Khoris and E. Y. Park, Plasmonic/magnetic molybdenum trioxide and graphitic carbon nitride quantum dots-based fluoroimmunosensing system for influenza virus, Sens. Actuators, B, 2020, 321, 128494.

55 L. A. A. Chunduri, A. Kurdekar, M. K. Haleyurgirisetty, E. P. Bulagonda, V. Kamisetti and I. K. Hewlett, Femtogram Level Sensitivity achieved by Surface Engineered Silica Nanoparticles in the Early Detection of HIV Infection, Sci. Rep., 2017, 7, 7149.

56 T. C. Chou, W. Hsu, C. H. Wang, Y. J. Chen and J. M. Fang, Rapid and specific influenza virus detection by functionalized magnetic nanoparticles and mass spectrometry, J. Nanobiotechnol., 2011, 9, 52.

57 M. Askaravi, S. E. Rezatofighi and S. Rastegarzadeh, Seifi Abad Shapouri MR: Development of a new method based on unmodified gold nanoparticles and peptide nucleic acids for detecting bovine viral diarrhea virus-RNA, $A M B$ Express, 2017, 7, 137.

58 S. R. Ahmed, J. Kim, T. Suzuki, J. Lee and E. Y. Park, Detection of influenza virus using peroxidase-mimic of gold nanoparticles, Biotechnol. Bioeng., 2016, 113, 2298-2303.

59 Y. Liu, L. Zhang, W. Wei, H. Zhao, Z. Zhou, Y. Zhang and S. Liu, Colorimetric detection of influenza A virus using antibody-functionalized gold nanoparticles, Analyst, 2015, 140, 3989-3995.

60 S. Oh, J. Kim, V. T. Tran, D. K. Lee, S. R. Ahmed, J. C. Hong, J. Lee, E. Y. Park and J. Lee, Magnetic Nanozyme-Linked Immunosorbent Assay for Ultrasensitive Influenza A Virus Detection, ACS Appl. Mater. Interfaces, 2018, 10, 12534-12543.

61 J. Lee, M. Morita, K. Takemura and E. Y. Park, A multifunctional gold/iron-oxide nanoparticle-CNT hybrid nanomaterial as virus DNA sensing platform, Biosens. Bioelectron., 2018, 102, 425-431.

62 H. W. Chen, Z. S. Fang, Y. T. Chen, Y. I. Chen, B. Y. Yao, J. Y. Cheng, C. Y. Chien, Y. C. Chang and C. M. J. Hu, Targeting and Enrichment of Viral Pathogen by Cell Membrane Cloaked Magnetic Nanoparticles for Enhanced Detection, ACS Appl. Mater. Interfaces, 2017, 9, 39953-39961.

63 S. R. Ahmed, K. Takemeura, T. C. Li, N. Kitamoto, T. Tanaka, T. Suzuki and E. Y. Park, Size-controlled preparation of peroxidase-like graphene-gold nanoparticle hybrids for the visible detection of norovirus-like particles, Biosens. Bioelectron., 2017, 87, 558-565.

64 S. Talebian, G. G. Wallace, A. Schroeder, F. Stellacci and J. Conde, Nanotechnology-based disinfectants and sensors for SARS-CoV-2, Nat. Nanotechnol., 2020, 15, 618-621. 
65 Reusable Graphene Mask Sterilises Itself against Coronavirus with Electrical Charge | STATNANO. [date unknown].

66 Nanotechnology in Battle Against Coronavirus | STATNANO. [date unknown].

67 New mask material can remove virus-size nanoparticles. [date unknown].

68 R. Itani, M. Tobaiqy and A. Al Faraj, Optimizing use of theranostic nanoparticles as a life-saving strategy for treating COVID-19 patients, Theranostics, 2020, 10, 5932-5942.

69 T. R. F. Smith, A. Patel, S. Ramos, D. Elwood, X. Zhu, J. Yan, E. N. Gary, S. N. Walker, K. Schultheis and M. Purwar, et al., Immunogenicity of a DNA vaccine candidate for COVID-19, Nat. Commun., 2020, 11, 1-13.

70 M. Lim, A. Z. M. Badruddoza, J. Firdous, M. Azad, A. Mannan, T. A. Al-Hilal, C. S. Cho and M. A. Islam, Engineered nanodelivery systems to improve dna vaccine technologies, Pharmaceutics, 2020, 12, 30.

71 Y. Abo-zeid, N. S. Ismail, G. R. McLean and N. M. Hamdy, A molecular docking study repurposes FDA approved iron oxide nanoparticles to treat and control COVID-19 infection, Eur. J. Pharm. Sci., 2020, 153, 105465.

72 J. S. Chahal, T. Fang, A. W. Woodham, O. F. Khan, J. Ling, D. G. Anderson and H. L. Ploegh, An RNA nanoparticle Vaccine against Zika virus elicits antibody and CD8+ T cell responses in a mouse model, Sci. Rep., 2017, 7, 252.

73 J. S. Chahal, O. F. Khan, C. L. Cooper, J. S. McPartlan, J. K. Tsosie, L. D. Tilley, S. M. Sidik, S. Lourido, R. Langer and S. Bavari, et al., Dendrimer-RNA nanoparticles generate protective immunity against lethal ebola, H1N1 influenza, and Toxoplasma gondii challenges with a single dose, Proc. Natl. Acad. Sci. U. S. A., 2016, 113, E4133-E4142.

74 L. Deng, T. Mohan, T. Z. Chang, G. X. Gonzalez, Y. Wang, Y. M. Kwon, S. M. Kang, R. W. Compans, J. A. Champion and B. Z. Wang, Double-layered protein nanoparticles induce broad protection against divergent influenza A viruses, Nat. Commun., 2018, 9, 359.

75 Y. Wang, G. L. Liu, D. L. Li, F. Ling, B. Zhu and G. X. Wang, The protective immunity against grass carp reovirus in grass carp induced by a DNA vaccination using singlewalled carbon nanotubes as delivery vehicles, Fish Shellfish Immunol., 2015, 47, 732-742.

76 B. Zhu, G. L. Liu, Y. X. Gong, F. Ling, L. S. Song and G. X. Wang, Single-walled carbon nanotubes as candidate recombinant subunit vaccine carrier for immunization of grass carp against grass carp reovirus, Fish Shellfish Immunol., 2014, 41, 279-293.

77 F. D. Cojocaru, D. Botezat, I. Gardikiotis, C. M. Uritu, G. Dodi, L. Trandafir, C. Rezus, E. Rezus, B. I. Tamba and C. T. Mihai, Nanomaterials designed for antiviral drug delivery transport across biological barriers, Pharmaceutics, 2020, 12, 171.

78 R. G. Kerry, S. Malik, Y. T. Redda, S. Sahoo, J. K. Patra and S. Majhi, Nano-based approach to combat emerging viral (NIPAH virus) infection, Nanomedicine, 2019, 18, 196-220.

79 M. Milovanovic, A. Arsenijevic, J. Milovanovic, T. Kanjevac and N. Arsenijevic: Nanoparticles in Antiviral Therapy, in
Antimicrobial Nanoarchitectonics: From Synthesis to Applications, Elsevier Inc., 2017:pp. 383-410.

80 H. H. Lara, E. N. Garza-Treviño, L. Ixtepan-Turrent and D. K. Singh, Silver nanoparticles are broad-spectrum bactericidal and virucidal compounds, J. Nanobiotechnol., 2011, 9, 30.

81 R. M. Mainardes and C. Diedrich, The potential role of nanomedicine on COVID-19 therapeutics, Ther. Delivery, 2020, 7, 411-414.

82 M. C. Sportelli, M. Izzi, E. A. Kukushkina, S. I. Hossain, R. A. Picca, N. Ditaranto and N. Cioff, Can nanotechnology and materials science help the fight against sars-cov-2?, Nanomaterials, 2020, 10, 802.

83 T. Y. Hu, M. Frieman and J. Wolfram, Insights from nanomedicine into chloroquine efficacy against COVID19, Nat. Nanotechnol., 2020, 15, 247-249.

84 C. N. Fries, E. J. Curvino, J.-L. Chen, S. R. Permar, G. G. Fouda and J. H. Collier, Advances in nanomaterial vaccine strategies to address infectious diseases impacting global health, Nat Nanotechnol, 2020, DOI: 10.1038/s41565020-0739-9.

85 S. Naseri, M. Darroudi, E. Aryan, A. Gholoobi, H. Rahimi, K. Ketabi, A. Movaqar, M. Abdoli, H. Gouklani and R. Teimourpour, et al., The Antiviral Effects of Curcumin Nanomicelles on the Attachment and Entry of Hepatitis C Virus, Iran J. Virol., 2017, 11, 29-35.

86 B. Mukherjee, B. Patra, B. Layek and A. Mukherjee, Sustained release of acyclovir from nano-liposomes and nanoniosomes: an in vitro study, Int. J. Nanomed., 2007, 2, 213-225.

87 S. H. Monavari, M. J. Mirzaei Parsa, B. Bolouri, S. A. Ebrahimi and A. Ataei-Pirkooh, The inhibitory effect of Acyclovir loaded nano-niosomes against herpes simplex virus type-1 in cell culture, Med. J. Islam Repub. Iran, 2014, 28, 99.

88 K. S. Joshy, S. S. Snigdha, N. Kalarikkal, L. A. Pothen and S. Thomas, Gelatin modified lipid nanoparticles for antiviral drug delivery, Chem. Phys. Lipids, 2017, 207, 24-37.

89 S. A. Loutfy, M. H. Elberry, K. Y. Farroh, H. T. Mohamed, A. A. Mohamed, E. B. Mohamed, A. H. I. Faraag and S. A. Mousa, Antiviral activity of chitosan nanoparticles encapsulating curcumin against hepatitis $\mathrm{C}$ virus genotype 4a in human hepatoma cell lines, Int. J. Nanomed., 2020, 15, 2699-2715.

90 V. Asgary, A. Shoari, M. Afshar Moayad, M. Shafiee Ardestani, R. Bigdeli, L. Ghazizadeh, M. S. Khosravy, E. Panahnejad, A. Janani and R. Bashar, et al., Evaluation of G2 Citric Acid-Based Dendrimer as an Adjuvant in Veterinary Rabies Vaccine, Viral. Immunol., 2018, 31, 47-54.

91 C. Lin, L. Chang, H. Chu, H. Lin, P. Chang, R. Y. L. Wang, B. Unnikrishnan, J. Mao, S. Chen and C. Huang, High Amplification of the Antiviral Activity of Curcumin through Transformation into Carbon Quantum Dots, Small, 2019, 15, 1902641.

92 T. Tong, H. Hu, J. Zhou, S. Deng, X. Zhang, W. Tang, L. Fang, S. Xiao and J. Liang, Glycyrrhizic-Acid-Based 
Carbon Dots with High Antiviral Activity by Multisite Inhibition Mechanisms, Small, 2020, 16, 1906206.

93 Z. Wang, T. Hu, R. Liang and M. Wei, Application of ZeroDimensional Nanomaterials in Biosensing, Front. Chem., 2020, 8, 320.

94 S. Manivannan and K. Ponnuchamy, Quantum dots as a promising agent to combat COVID-19, Appl. Organomet. Chem., 2020, 34, e5887.

95 V. Lysenko, V. Lozovski and M. Spivak, Nanophysics and antiviral therapy, Ukr. J. Phys., 2013, 58, 77-90.

96 V. Lozovski, V. Lysenko, V. Piatnytsia, O. Scherbakov, N. Zholobak and M. Spivak, Physical point of view for antiviral effect caused by the interaction between the viruses and nanoparticles, J. Bionanosci., 2012, 6, 109-112.

97 J. A. Jackman, P.-Y. Shi and N.-J. Cho, Targeting the Achilles Heel of Mosquito-Borne Viruses for Antiviral Therapy, Nat. Mater., 2018, 17, 955-956.

98 B. K. Yoon, W.-Y. Y. Jeon, T. N. Sut, N.-J. J. Cho and J. A. Jackman, Stopping Membrane-Enveloped Viruses with Nanotechnology Strategies: Toward Antiviral Drug Development and Pandemic Preparedness, ACS Nano, 2021, 15, 125-148.

99 J. Münch, E. Sanchez-Garcia, J. Shorter, T. Schrader, T. Weil, R. Diger Groß, A. Röcker, K. Bravo-Rodriguez, C. Heid and A. Sowislok, et al., Supramolecular mechanism of viral envelope disruption by molecular tweezers, J. Am. Chem. Soc., 2020, 142, 17024-17038.

100 F. Vigant, N. C. Santos and B. Lee, Broad-spectrum antivirals against viral fusion, Nat. Rev. Microbiol., 2015, 13, 426-437.
101 J. Zou and P. Y. Shi, Targeting vesicle size, Nat. Mater., 2018, 17, 955-956.

102 D. Ting, N. Dong, L. Fang, J. Lu, J. Bi, S. Xiao and H. Han, Multisite inhibitors for enteric coronavirus: antiviral cationic carbon dots based on curcumin, ACS Appl. Nano Mater., 2018, 1, 5451-5459.

103 X. Dong, M. M. Moyer, F. Yang, Y. P. Sun and L. Yang, Carbon Dots' Antiviral Functions Against Noroviruses, Sci. Rep., 2017, 7, 519.

104 L. Chen and J. Liang, An overview of functional nanoparticles as novel emerging antiviral therapeutic agents, Mater. Sci. Eng., C, 2020, 112, 110924.

105 T. Du, J. Liang, N. Dong, L. Liu, L. Fang, S. Xiao and H. Han, Carbon dots as inhibitors of virus by activation of type I interferon response, Carbon, 2016, 110, 278-285.

106 V. Lozovski, V. Lysenko, V. Pyatnitsia and M. Spivak, Can nanoparticles be useful for antiviral therapy?, Semicond. Phys., Quantum Electron. Optoelectron., 2011, 14, 489-491.

107 D. Chauhan, R. Prasad, R. Srivastava, M. Jaggi, S. Chauhan and M. Yallapu, Comprehensive Review on Current Interventions, Diagnostic, and Nanotechnology Perspectives against SARS-CoV-2, Bioconjugate Chem., 2020, 31, 2021-2045.

108 H. Chibli, L. Carlini, S. Park, N. M. Dimitrijevic and J. L. Nadeau, Cytotoxicity of InP/ZnS quantum dots related to reactive oxygen species generation, Nanoscale, 2011, 3, 2552-2559.

109 M. Cotrim and R. Oréfice, Biocompatible and fluorescent polycaprolactone/silk electrospun nanofiber yarns loaded with carbon quantum dots for biotextiles, Polym. Adv. Technol., 2021, 32, 87-96. 